\title{
Developing a Fire Monitoring and Control System Based on IoT
}

\author{
$\mathrm{Yi} \mathrm{Li}^{1}$, Jianjun $\mathrm{Yi}^{2, *}$, Xiaoming Zhu ${ }^{3}$, Zhuoran Wang ${ }^{4}$ and Fangwen $\mathrm{Xu}^{5}$ \\ Department of Mechanical Engineering, East China University of Science and Technology, Shanghai, China \\ ${ }^{*}$ Corresponding author
}

\begin{abstract}
An novel fire extinguishing monitoring and control system is proposed in this paper. The system combines online monitoring of fire extinguishing information based on Internet of things (IoT), early fire warning and alarm system with building safety evaluation, which are designed to acquiring the real-time information of fire extinguishing working condition, improving the reliable prediction mechanism in traditional fire monitoring system. A perception terminal based on IoT is developed and the fire real-time monitoring and control system is designed in this paper. The novel system can acquire the real-time working condition information of fire extinguishing facilities, such as pipe flow, pressure, temperature and humidity of the environment, current and voltage of the electric equipment, valve switch, relay action, alarming message, it can upload the collected data to the monitoring center through the $\mathrm{C} / \mathrm{S}$ (Client /Server) architecture. Based on these data, an electrical early fire warning model based on BP neural network is proposed. A fire safety evaluation model based on ontology with the usage of protege is established, which facilitate the on-line monitoring and control of building fire protection information, the prediction of electrical fire and improve building safety level evaluation.
\end{abstract}

Keywords-internet of things(IoT); fire monitoring and control; ontology; fire safety evaluation

\section{INTRODUCTION}

With the rapid development of China's economic construction, intensive increasing of urban population and lasting expansion of city building density, underground engineering, high-rise buildings and large public constructions become more and more, objectively it puts forward more severe challenge to urban fire protection. In order to adapt to the modern city and the public safety of society development, the real time remote-monitoring system of urban fire protection based on IoT is proposed in this paper as a solution to solve the above problem.

The design and implementation of monitoring products for the fire protection system based on IoT is introduced in detail in this paper. The IoT terminal products are responsible for acquiring the working status of the fire extinguishing and protection system, such as the building fire facilities alarm, hydrant pipe flow, environmental temperature, etc. Through IoT, effective sensing, reliable transmission and centralized and efficient management of the fire system can be available.

\section{AN OVERALL FRAMEWORK OF FIRE MONITORING INFORMATION SYSTEM BASED ON IOT}

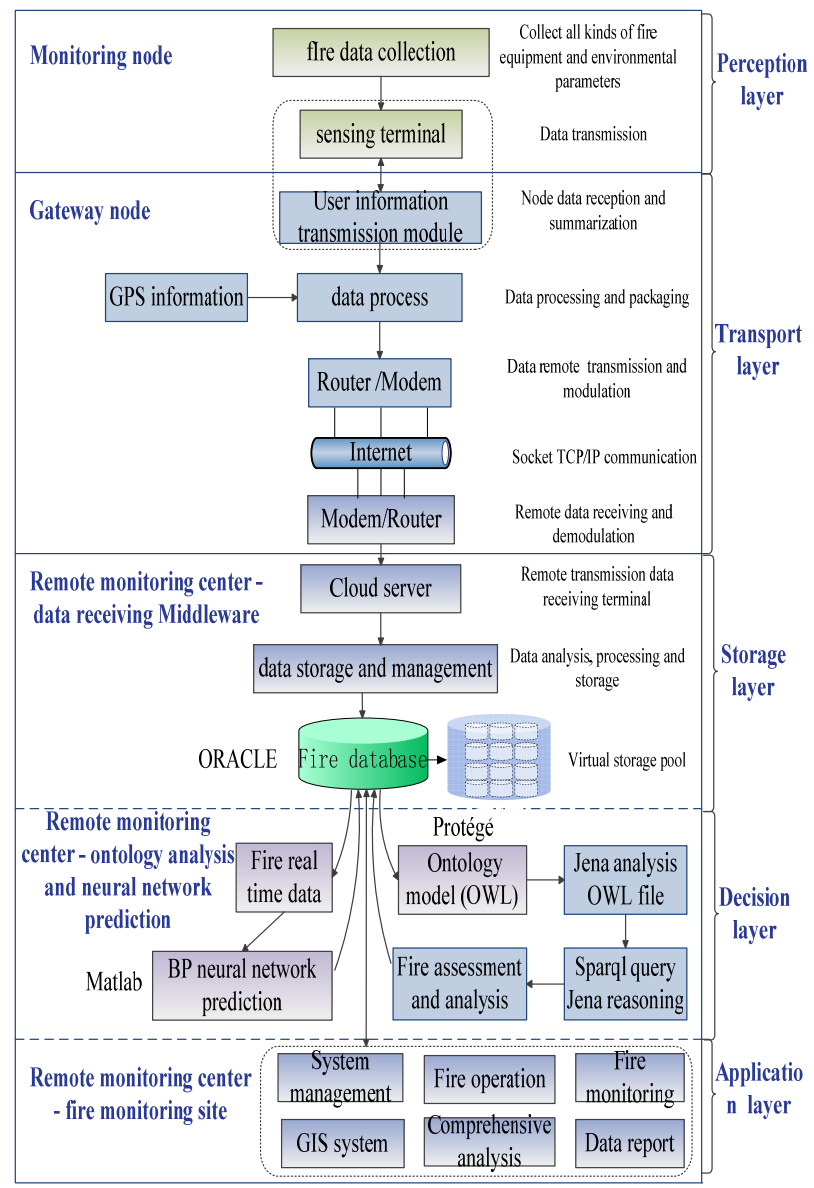

FIGURE I. MONITORING INFORMATION SYSTEM ARCHITECTURE BASED ON IOT

The framework of fire monitoring information system which is shown in Figure I consists of 5 architecture layers: the perception layer, transport layer, storage layer, intelligent decision-making layer and application layer. The developed monitoring terminal module can collect the information of realtime alarm of fire control cabinet, current and voltage of electrical equipment, pipeline flow and pressure, ambient temperature and humidity, valves switch, relay action with the set frequency. The collected data including the node number and time stamp will be packed in accordance with the 
agreement that is already made by the system before it is uploaded to the user information transfer module through the WIFI or CAN bus network. At the same time the camera inside the building may upload the collected graphic information to the user information transmission module through the local network. RFID fire equipment inventory operation and maintenance subsystem is included in the system and the inventory or maintenance information can be transmitted to the user information transmission module through the mobile RFID handheld terminal or the USB connection port. In the system, the user information transmission module is equivalent to the gateway which summarizes all the monitoring information in the perception layer.

In transport layer we developed the $\mathrm{C} / \mathrm{S}$ data transmission software based on TCP / IP and windows platform. In order to ensure the safety and reliability of the data transmission, RSA+AES double encryption is applied in data transmission. At the same time, the client has the function of the reconnection of broken network and the retransmission of temporary data. The sensor value, the video image as well as the RFID information in accordance with the TCP/IP are packed, loaded with the GPS stamp, and then are uploaded to the cloud server through the router and internet from the user information transmission module.

Efficient ORALCE in storage layer is served as a storage database. The cloud server receives the fire monitoring information of the buildings through internet transmission and provides data support for the following intelligent decision and application layer. Meanwhile we adopt the technology of mature load balance, double hot standby and other technologies to ensure the safety and reliability of data storage, virtual storage technology to ensure the flexible expansion of data capacity.

In intelligent decision-making layer, the fire monitoring alarm and fire safety evaluation concerning the collected data are accomplised. By fire alarm it means the acquisition to the use of electrical equipment leakage current, working voltage, working current and cable temperature values as input to produce the fire probability by using $\mathrm{BP}$ neural network algorithm. Fire safety evaluation is about ontology modeling, ontology analysis and reasoning based on fire information collected by the building to give fire safety evaluation of the monitoring building.

In the application layer the interaction between the monitoring system and the user is mainly realized. Management staff can see the building's real-time location, pipe flow and pressure, temperature and humidity of the environment, current and voltage of the electric equipment, valve switch, relay, fire control cabinet real-time alarm information, operation and maintenance information, historical data, the building fire alarm and fire safety evaluation information through the website as well as various data reports. In addition, monitoring site also covers the two modules: the user management and site management. The former is convenient for management personnel to add and delete users, modify user information, and manage user rights etc. While the latter is mainly about the management of each monitoring site, including basic information about the site of additions and deletions, threshold settings of site alarms as well as the addition and query of site maintenance logs.

\section{The Main TEChNOLOgy ReALIZATION OF FIRE MONITORING INFORMATION SYSTEM BASED ON IOT}

A. Implementation of Fire Perception Layer Architecture Based on IoT

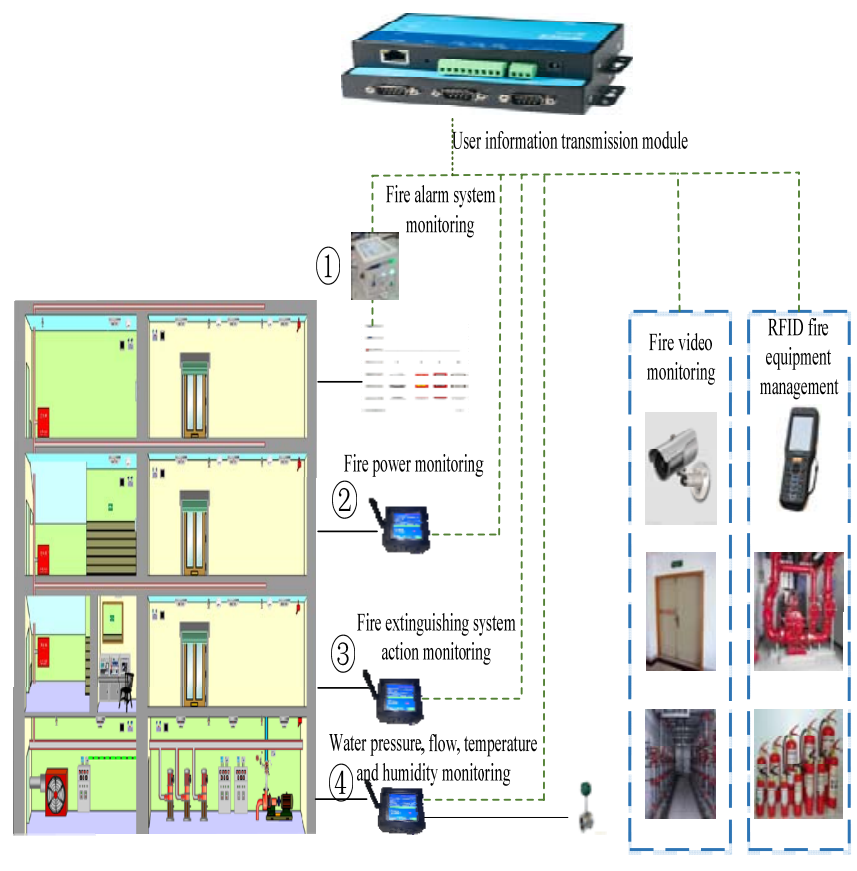

FIGURE II. FIRE MONITORING INFORMATION PERCEPTION SCHEMATIC BASED ON IOT

As shown in Figure II. In this system, (1) (2) (3) (4) is the perception terminal compatible with 232/485/CAN/WIFI and other means of communication. It also has touch screen, The communication rate, the sampling frequency, the node number and the network connection parameters of the module can be set online, and the set parameters are stored in the outer FLASH, and the power up again is not required to be repeated:

Module (1) access fire control cabinet inside buildings through 232 bus, and receive temperature and smoke, sound and light alarm by interrupt and finally spread to local user information transmission module through WiFi or Ethernet. Module (2) achieve the online monitoring of fire protection equipment's voltage, current indicators. The data collected along with the node number is packed in accordance with the CAN bus protocol, then upload to the user information transfer module. Module (3) is mainly to achieve the on-line monitoring of fire shutter, relay and pipeline valve. When the switch action of the monitored equipment generated interrupt, the module uploads these status to the user information module according to the protocol of CAN bus. Module (4)mainly realizes the online monitoring of water pressure, flow, temperature and humidity, and other sensing monitor.

At the same time the sensing terminal also includes fire video surveillance and mobile RFID monitoring terminal, here 
we use Hikvision 720p industrial grade cameras to monitor important fire control facilities and fire channel. At the same time, the development of fire equipment inspection and maintenance system based on Phone Windows is developed. So far, perception layer all data will eventually converge to the user information transmission module, then the data realize local storage and display and eventually upload to the cloud server through the Internet.

\section{B. Perception Layer Protocol Composition Based on IoT}

From the above we can know, the sensing terminal based on IoT collect power indicators, sensing value, shutter doors and other fire protection equipment's state of action in real-time, finally to upload the data through the CAN bus. Here according to the standard CAN data frame, we make the CAN bus data protocol for the data transmission of the system.

The data segment's total length is 64 bit eight bytes, specific protocols such as table shown in the 1 . The first high byte retention, the low byte is monitoring type, second byte on behalf of the floor, and 3 to 8 bytes respectively represent the node number and the specific value.

TABLE I. SENSOR DATA SEGMENT

\begin{tabular}{|c|c|c|c|c|}
\hline Type & Floor & $\begin{array}{l}\text { Node number } \\
\text { pressure/flow/temperature } \\
\text { (MPa } \backslash \text { 3/s } \backslash \text { degrees C) } \\
\end{array}$ & $\begin{array}{l}\text { Retai } \\
\text { n }\end{array}$ & $\begin{array}{l}\text { Retai } \\
\text { n }\end{array}$ \\
\hline $0 x f 4$ & $\begin{array}{l}\text { 0x00- } \\
\text { 0xff }\end{array}$ & $\begin{array}{llll}\text { 0xy } & 0 x m z & 0 x m & 0 x f n \\
z & & n & \\
\end{array}$ & $0 x f f$ & 0xff \\
\hline $\begin{array}{l}\text { f: Retain } \\
\text { low4: } \\
\text { pressure . } \\
\text { etc }\end{array}$ & $\begin{array}{l}0-255 \\
\text { layer }\end{array}$ & $\begin{array}{l}\text { Node Number: ymm(0-255) } \\
\text { (dial switch setting) } \\
\text { Senor Value: zz.nn(0-99.99) }\end{array}$ & & \\
\hline
\end{tabular}

\section{Fire Safety Evaluation Based on Ontology}

Because the building information we get is heterogeneous, different buildings should be installed different end perception systems, similarly different fire operation and maintenance personnel record different styles of operation and maintenance information. Ontology makes such model of knowledge possible in order that these heterogeneous data consisting of basic information, maintenance, alarm and terminal sensing information of the monitored construction could be effectively organized. Ontology, an informational technological term which is developed in recent years, serves as the basis for the Semantic Web and artificial intelligence. It is a shared formal description on the important concepts of a specific domain (fensel, 2001) [1].It can realize the integration of heterogeneous data, and can eliminate the ambiguity of the expressions of the concepts [2].The efficiency of the search, accumulation, and sharing of knowledge will be greatly improved with the support of ontology. Furthermore it makes the reuse and sharing of knowledge in a real sense has become possible (Panetto, and Tursi Dassisti, 2012) [3-5].Owing to the above-mentioned properties and advantages, the technology of ontology is used in this paper to process semantically the overall information of the fire monitoring buildings, maintenance, alarm, the situation of fire equipment, temperature and humidity, the flow of pipeline etc. Also we establish ontology model, combined with the cloud server, an ontology instance is formed. With what has been discussed above as a foundation, the inference rules are established, and the final result is gained by the inference engine.

There are various ontologies, which can be divided into general, domain, representation and task ontology. The fire monitoring information ontology model at the Internet of things in this paper is considered as the domain ontology, While Protege 3.3 is chosen as the ontology modeling tool.

Class is one of the cores of ontology model. It is used to describe the concept involved in the field, and it is an example of set degree. The top-down approach is applied in this paper, that is to say that a fire information model is established first and subsequently each sub segment is subdivided by layer, until the final completion of the entire class is established. The sub category of the fire information in this paper includes: information and evaluation, the secondary subclasses that belong to evaluation are marked as I, II, III, IV, V. While the following 11 secondary sub classes consisting of building ,time, inspection and maintenance records, fire, video monitoring, automatic alarm, supply voltage, temperature, humidity, pipe flow, pipe pressure are classified into the category of information.

Herein, the class of ontology needs to be described after the completion of the establishment. Those specific data of attributes and constraints given to the second sub classes include the name, address, latitude and longitude, type, property owners etc.

Thus we have completed the establishment of the model of fire information system based on IoT. We can get a tree-like figure through the owl Viz editor with protégé. It is shown in Figure III below:

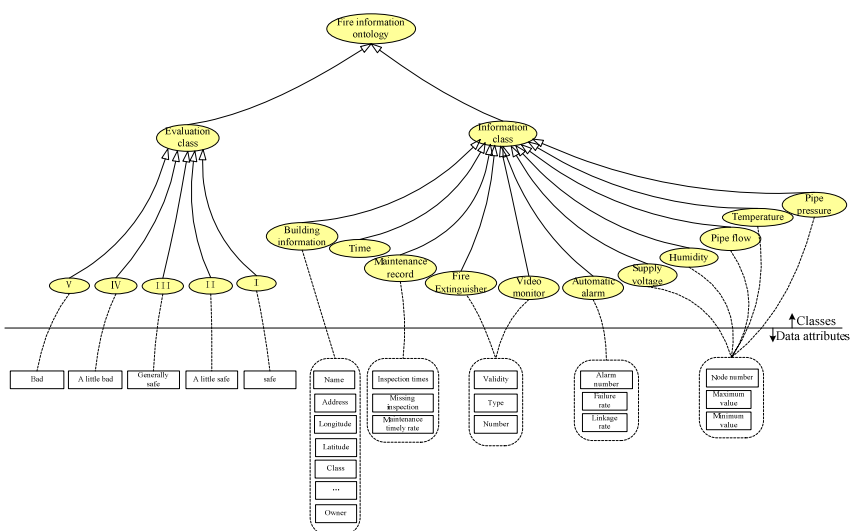

FIGURE III. THE ONTOLOGY MODEL OF FIRE MONITORING INFORMATION BASED ON IOT

The above-discussed construction of the model of the fire ontology information is a prerequisite for the realization of the fire ontology analysis. It is necessary to develop the relevant application to analyze and operate the ontology model. The C\# developed background application software is applied in the ontology analysis module on the fire information system based on IoT. The whole process is like this: the classes of Java and the package of Jena are introduced into the Studio Visual 2008 platform and the fire information ontology is approched into the C\# environment. Next, the evaluation index numerical information can be inquired through a enquiry language called 
Sparql. Subsequently the fire information is evaluated by the comprehensive evaluation method, and finally the building's fire safety level and the security measures that should be taken by the fire management personnel through the application of the reasoning machine Jena are analyzed and evaluated .The specific implementation process is shown in figure 4:

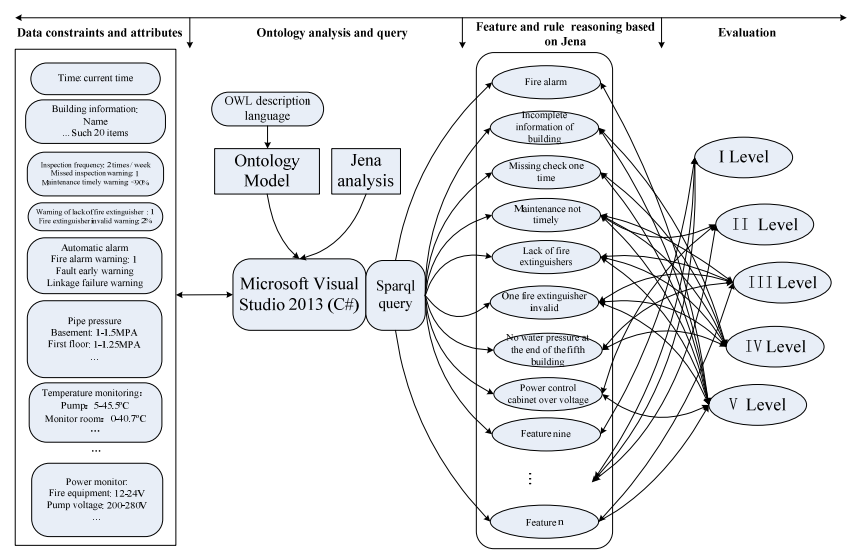

FIGURE IV. SEMANTIC PROCESSING AND QUERY OF FIRE MONITORING SECURITY EVALUATION BASED ON IOT

\section{THE VERIFICATION OF FiRE INFORMATION SYSTEM BASED ON IOT}

As shown in Figure V, in this paper, our self-developed terminal server based on IoT was installed in the building of Hunan Mass Media Broadcasting Company to collect the leakage current, voltage, current, pressure pipes, pipe flow of the charged equipment and the fire alarm information of the panel in the building, etc. Then the information is encrypted through the C \# data Client based on AES that we developed, subsequently it is transmitted to Server on the Internet. The data receiving Server which runs on the server end receives the data to decrypt them, and then they are parsed and stored in the Oracle database, which is used for decision making and application.

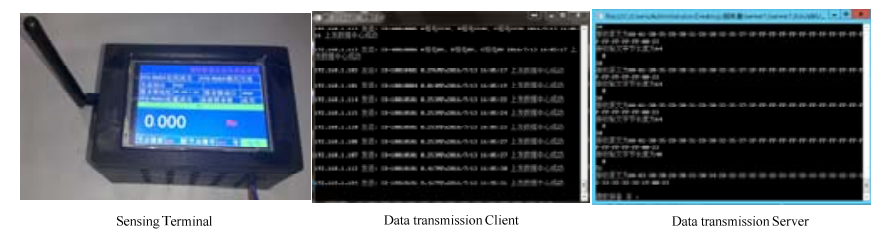

FIGURE V. FIRE MONITORING AND SENSING TERMINAL BASED ON IOT AND C/S

What is displayed with Figure VI is the online monitoring site run on the server side. That shows part of the current monitoring information of the building. The results shown in Figure VII which is verified to be equivalent with the real situation are obtained from the electrical fire prediction of $\mathrm{BP}$ neural network and the fire safety assessment of the buildings grounded on ontology model based on the collected information shown in Figure VI. To sum up, the previouslymentioned fire safety evaluation system on the ground of ontology and the electrical fire prediction related with $\mathrm{BP}$ neural network is so stable and accurate that they can meet the requirements of fire alarm and safety assessment.

\begin{tabular}{|c|c|c|c|c|}
\hline \multicolumn{4}{|c|}{ Monitoring System Based on IoT } & 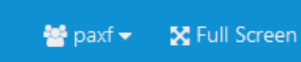 \\
\hline NODE ID & MONITORING TYPES & STATUS & POSITION & TIME \\
\hline 1001001 & leakage current & $0.011 \mathrm{~A}$ & No.1 power of rain-pump in control cabinet of pump station & 2016/7/13 11:34:00 \\
\hline 1001001 & operating current & $2.17 \mathrm{~A}$ & No.1 power of rain-pump in control cabinet of pump station & 2016/7/13 11:34:00 \\
\hline 1001001 & working voltage & $222 \mathrm{~V}$ & No.1 power of rain-pump in control cabinet of pump station & 2016/7/13 13:34:00 \\
\hline 1001001 & cable temperature & $40^{\circ} \mathrm{C}$ & No.1 power of rain-pump in control cabinet of pump station & 2016/7/13 11:34:00 \\
\hline 1001008 & leakage current & $0.23 \mathrm{~A}$ & No.1 fan control cabinet outside pump station & $2016 / 7 / 13$ 14:34:00 \\
\hline 1001008 & operating current & $2.01 \mathrm{~A}$ & No.1 fan control cabinet outside pump station & $2016 / 7 / 1314: 34: 00$ \\
\hline 1001008 & working voltage & $220 \mathrm{~V}$ & No. 1 fan control cabinet outside pump station & 2016/7/13 13:34:00 \\
\hline 1001008 & cable temperature & $48^{\circ} \mathrm{C}$ & No.1 fan control cabinet outside pump station & $2016 / 7 / 13$ 14:34:00 \\
\hline 2001193 & fire smoke & alarm & after the studio & $2016 / 7 / 13$ 14:34:08 \\
\hline 3003004 & pipe pressure & $0.0038 \mathrm{MPa}$ & spray end on the third floor of the Southeast & $2016 / 7 / 13$ 14:35:08 \\
\hline 3001005 & pipe pressure & $0.1 \mathrm{MPa}$ & Water cannon pump in underground water pump station & 2016/7/13 14:35:08 \\
\hline 4001005 & humidity & $40 \%$ RH & underground water pump station & 2016/7/13 15:33:08 \\
\hline 4001018 & humidity & $20 \%$ RH & monitoring room on the second floor & 2016/7/13 15:35:08 \\
\hline
\end{tabular}

FIGURE VI. FIRE REAL TIME MONITORING DATA BASED ON IOT 


\begin{tabular}{|c|c|c|c|}
\hline \multicolumn{2}{|c|}{ Fire Prediction of BP Netural Network } & \multicolumn{2}{|c|}{ Ontology Security Evaluation } \\
\hline NODE ID & FIRE PROBABILITY & \multirow{2}{*}{$\begin{array}{l}\text { CURRENT BUILDING } \\
\text { LEVEL : }\end{array}$} & \multirow[b]{2}{*}{ v } \\
\hline 1001001 & 0.194 & & \\
\hline 1001008 & 0.762 & \multicolumn{2}{|c|}{ DETAILED ASSESSMENT } \\
\hline & & Name & $\begin{array}{l}\text { Studio Floor of DaZhongChuanMei in HuNan } \\
\text { province }\end{array}$ \\
\hline & & Time & 2016/7/13 11:34:00 \\
\hline & & Basic Information Perfect Degree & Good \\
\hline & & Alarm Information & one smoke alarm \\
\hline & & Fire Extinguisher & Failure one \\
\hline & & Inspection Information & Good \\
\hline & & Pipe pressure & Nomal \\
\hline & & Ambient Temperature & Good \\
\hline
\end{tabular}

FIGURE VII. THE RESULTS OF BP NEURAL NETWORK FIRE PREDICTION AND ONTOLOGY EVALUATION BASED ON IOT

\section{CONCLUSION}

In this paper, the architecture and implementation of fire control system based on IoT is discussed. Furthermore, the safety evaluation system based on ontology is built, that is to say the realization of the fire information reasoning and evaluation is accomplished. So that the fire regulators and users can obtain visually the accurate fire monitoring information on early and the results of fire evaluation of the building. Therefore the reliability of early fire warning and the effectiveness of supervision are improved. Due to its simple operation, flexible application and high stability, the proposed fire-based information system based on IoT can be widely used as on-line monitoring, fire alarm and fire safety evaluation in large public places, ancient buildings and forest fires.

\section{ACKNOWLEDGMENTS}

This paper was supported by the Research Foundation of Science and Technology Commission of Shanghai under Grant No. 10DZ1500200, the Natural Science Fund of China (NSFC) under Grant Nos. 50975088, 51275173, 51575186 and 51210105018, the Fundamental Research Funds for the Central Universities under Grant No. WH0913009, Shanghai Pujiang Program under Grant No. PJ201000353, and Shanghai Software and IC industry Development Special Fund under Grant No. 120493.

\section{REFERENCES}

[1] Fensel D. Ontologies: A silver bullet for knowledge management and electronic commerce[M]. Berlin: Spring-Verlag, 2001.

[2] Borst WN.Construction of engineering ontologies for knowledge sharing and reuse[D]. Universiteit Twente, 1997.

[3] Chira O, Chira C, Roche T, Tormey D, Brennan A. An agent-based approach to knowledge management in distributed design[J]. Journal of Intelligent Manufacturing.2006, 17(6): 737-750.

[4] Panetto H, Dassisti M, Tursi A. ONTO-PDM: product-driven ONTOlogy for Product Data Management interoperability within manufacturing process environment[J].Advanced Engineering Informatics. 2012, 26(2): 334-348.
[5] Imran M, Young B. The application of common logic based formal ontologies toassembly knowledge sharing[J]. Journal of Intelligent Manufacturing. 2015, 26(1): 139-158. 